\title{
EXPERIENCE IN THE FUNCTIONING OF THE STROKE UNIT IN THE STRUCTURE OF THE UNIVERSITY CLINIC OF THE ODESSA NATIONAL MEDICAL UNIVERSITY
}

Y. Vorokhta ${ }^{1}$, T. Muratova ${ }^{1}$, D. Khramtsov ${ }^{1}$, O. Stoyanov ${ }^{2}, \mathrm{M}$. Vikarenko ${ }^{1}$, G. Kozlova ${ }^{1}$, I. Dobush ${ }^{1}$, E. Andrushchenko ${ }^{1}$.

${ }^{1}$ University Clinic, Neurology, Odessa, Ukraine.

${ }^{2}$ Odessa National Medical University, Neurology, Odessa, Ukraine.

Background and Aims. The stroke unit of the university clinic has been functioning since April 4, 2016. It is organized on the basis of the neurological department of the clinic and includes an intensive monitoring ward and early recovery wards equipped with vital and video monitoring systems and the necessary paraphernalia. This study was aimed to assess the experience of stroke unit work in 2016-2017. Method: Official reports and medical records for the period of 20162017 were analysed.

Results. Patient care is carried out on the principle of a multidisciplinary team, with the participation of a neurologist, cardiologist, therapist, neurosurgeon, vascular surgeion, rehabilitationist, speech therapist, neuropsychologist, ergotherapist. Interaction of the hospital units allows revascularization by mechanical thrombectomy, stenting or carotid endarterectomy. The clinic's stroke unit takes part in international projects such as ESO-EAST, Angels, SITSEAST, CHRONEX. On the basis of the stroke unit interns from European universities as well as interns and graduate students of ONMedU can improve their skills and competence.

During the stroke center work, 167 patients with acute stroke were treated, including 154 with ischemic stroke, and 13 with hemorrhagic stroke. Post-stroke partial seizures were registered in 7 (4.2\%) patients, generalized seizure by type of absence - in one (0.6\%) case. Mortality rate was $9.0 \%$. Satisfactory functional results were achieved in $83.2 \%$ of patients.

Conclusion The organization and development of the stroke unit in the structure of the university clinic makes it possible to optimize the provision of emergency medical care to patients with acute disorders of cerebral circulation.

Keywords: stroke unit, university clinic, medical education, multidisciplinary team 